\title{
UV Emission in Type Ia Supernova Elliptical Host Galaxies
}

\author{
Brad E. Tucker \\ Mt. Stromlo Observatory, via Cotter Road, \\ Weston Creek, ACT 2611 Australia \\ email: brad@mso.anu.edu.au
}

The current use of Type Ia supernova (SN Ia) as standard candles is to measure the dark energy equation-of-state to better than $10 \%$. However, we still lack a clear understanding of their progenitor systems. We analyze the host galaxies of type Ia Supernova (SN Ia) discovered by the ESSENCE survey using UV and optical data, as studying the environments of SN Ia is a great way to understand the progenitors. We developed a new method for determining the SED and rest-frame magnitudes of the host galaxies and we use empirical relations to derive stellar mass and star-formation rate (SFR) measurements of the host galaxies. We find a high rate of UV emission in our passive galaxies, suggesting current star-formation in these galaxies. Specifically, we have found that at $z>0.3, \approx 60 \%$ of the elliptical host galaxies have star-formation while with nearby hosts, it is $\approx 40 \%$, with both samples having an additional $\approx 10-15 \%$ of hosts being AGN. We also find a connection between the time required for the progenitor to evolve and explode as an SN and UV emission in the elliptical hosts, suggesting that UV emission in elliptical galaxies is indeed star-formation and linked to the production of SN Ia's.

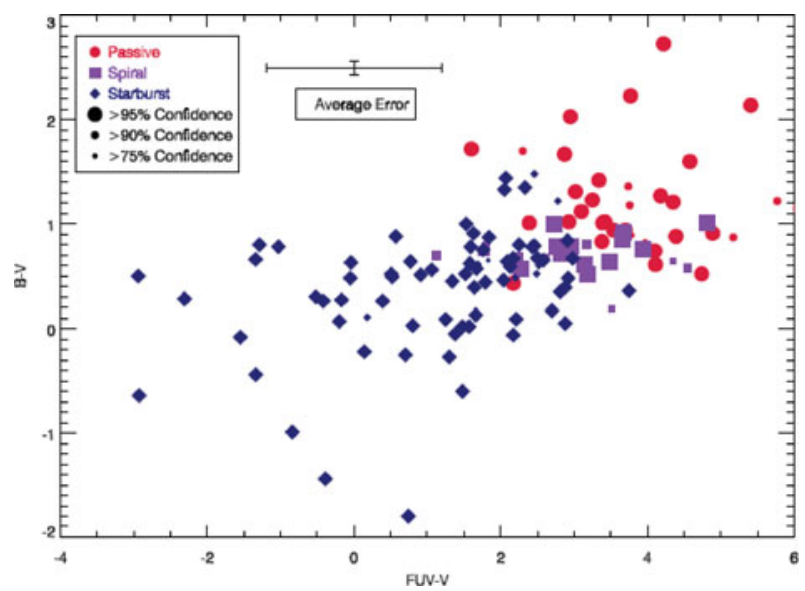

Figure 1. The rest-frame $F U V-V$ and $B-V$ colors of the host galaxies in the ESSENCE survey from Tucker et al. (2012a). The red circles are passive galaxies, purple squares are spiral galaxies, and the blue diamonds are active galaxies. The size of the point represents the confidence in the fit, with the largest points having a fit $>95 \%$, the middle-size points $90 \%<$ fit $<95 \%$, and the smallest points $75 \%<$ fit $<90 \%$. We also show the corresponding average error-bar in the top..

\section{References}

Tucker, B. E., et al. 2012a, ApJ, submitted

Tucker, B. E., et al. 2012b, ApJ, submitted 